\title{
Hard sphere colloidal dispersions: Mechanical relaxation pertaining to thermodynamic forces
}

\author{
J. Mellema, C. G. de Kruif, C. Blom, and A. Vrij \\ Rheology Group, Department of Applied Physics, Twente University of Technology, Enschede and \\ Van't Hoff Laboratory for Physical and Colloidal Chemistry, University of Utrecht (The Netherlands)
}

\begin{abstract}
The complex viscosity of sterically stabilized (hard) silica spheres in cyclohexane has been measured between $80 \mathrm{~Hz}$ and $170 \mathrm{kHz}$ with torsion pendulums and a nickel tube resonator. The observed relaxation behaviour can be attributed to the interplay of hydrodynamic and thermodynamic forces. The validity of the Cox-Merz rule is checked.
\end{abstract}

Key words: Hard sphere, complex viscosity, linear viscoelasticity, thermodynamic force, Cox-Merz rule

\section{Introduction}

When the macroscopic stress tensor is expressed in terms of microscopic variables it can often explain phenomena observed in static, steady-state and dynamic experiments. To deduce the stress tensor of dispersions one has to take into account the dynamics of intermolecular and interparticle forces, the hydrodynamic forces between particles and ambient fluid and the conservation equations governing the spatial and oriental distribution of particles and molecules.

A better understanding of dispersions, which are rheologically extremely complex, can be obtained from theoretical and experimental studies in which one or more of the following aspects dominate: strong Van der Waals interactions [1], elasticity of long range electrostatic repulsions [2-6], and long range steric interaction in suspensions at volume fraction approaching closest packing [7-9]. In hard sphere suspensions hydrodynamic, Brownian and hard sphere interactions [10, 11] dominate, whereas in emulsions interface effects are important $[12,13]$.

In previous studies $[10,11]$, by the results of static and steady-state experiments, evidence has accumulated that sterically stabilized silica spheres behave as hard spheres. In the present paper the study of that system is continued with linear dynamic experiments. In the following section it is hypothesized that such hard spheres display relaxation phenomena of hydrodynamic and thermodynamic forces. The experimental aspects of this study are elucidated in section 3 . The final section contains the results and discussion, it is concluded that the predicted relaxation phenomena actually exist.

\section{Theoretical predictions}

The deviatoric part of the macroscopic stress tensor $\mathbf{T}$ for a dispersion of hard particles suspended in a Newtonian fluid with viscosity $\eta_{0}$ in a flow field with rate of strain tensor $\dot{\mathbf{E}}$ can be given by [14]

$$
\mathbf{T}=2 \eta_{0} \dot{\mathbf{E}}+\frac{1}{V} \sum_{i} \mathbf{S}_{i}-\frac{1}{V} \sum_{i} \boldsymbol{x}_{i} \boldsymbol{F}_{i}+\frac{1}{V} \frac{1}{3}\left(\sum_{i} \boldsymbol{x}_{i} \cdot \boldsymbol{F}_{i}\right) \mathbf{1} .
$$

In (1) the stresslet $\mathbf{S}_{i}$ and $\boldsymbol{x}_{i} \boldsymbol{F}_{i}$ are averaged over a volume $V$. The stresslet $\mathbf{S}_{i}$ of a particle at position $x_{i}$ is given by an integral over its surface $A_{i}$

$$
\mathbf{S}_{i}=\int_{A_{i}}\left\{\left(\boldsymbol{x}-\boldsymbol{x}_{i}\right) \boldsymbol{\sigma} \cdot \boldsymbol{n}-\frac{1}{3}\left[\left(\boldsymbol{x}-\boldsymbol{x}_{i}\right) \cdot \boldsymbol{\sigma} \cdot \boldsymbol{n}\right] \mathbf{1}\right\} d A_{i}
$$

where $\boldsymbol{x}$ is the coordinate of a point at the surface of a particle, $\boldsymbol{n}$ is the normal at that point pointing outwards and $\boldsymbol{\sigma}$ is the stress exerted by the fluid on the surface. The force $\boldsymbol{F}_{i}$ experienced by a particle at position $\boldsymbol{x}_{i}$ is given by [14]

$$
F_{i}=-\nabla_{i} U-k T \nabla_{i} \ln P_{N}
$$

where $U$ is the interaction potential and $P_{N}$ is the probability density of the $N$ particles in volume $V$, of 
which $P_{N} d x_{1}, \ldots, d x_{N}$ gives the probability of finding $N$ spheres at positions $\boldsymbol{x}_{1}, \ldots, \boldsymbol{x}_{N}$. The function $P_{N}$ satisfies the conservation relation

$$
\frac{\partial P_{N}}{\partial t}+\sum_{i=1}^{N} \nabla_{i} \cdot \dot{j}_{i}=0
$$

in which flux $\boldsymbol{j}_{i}$ is given by

$$
j_{i}=P_{N}\left(\boldsymbol{u}_{i}+\sum_{j=1}^{N} \boldsymbol{\omega}_{i j} \cdot \boldsymbol{F}_{j}\right)
$$

The velocity $\boldsymbol{u}_{i}$ and the hydrodynamic mobility $\boldsymbol{\omega}_{i j}$ are dependent on the configuration of the $N$ particles.

For reasons of simplicity the calculations involve only the two-body distribution function which at equilibrium (superscript 0 ) is related to the radial distribution function $g(r)$ by

$$
P_{2}^{0}\left(\boldsymbol{x}_{1}, \boldsymbol{x}_{2}\right)=n^{2} g(r)
$$

where $n$ is the number density and $r=\left|\boldsymbol{x}_{1}-\boldsymbol{x}_{2}\right|$.

The conditions of linearity requires that

$$
\dot{E} \equiv(\dot{\mathbf{E}}: \dot{\mathbf{E}})^{1 / 2} \rightarrow 0
$$

For spheres with radius $a$ in an ambient fluid with viscosity $\eta_{0}$ this condition is satisfied if for the Péclet number $P e$ the inequality

$$
P e=\frac{\eta_{0} a^{3}}{k T} \dot{E} \equiv \frac{1}{6 \pi} \frac{a^{2} \dot{E}}{D_{0}} \ll 1
$$

holds. If the Reynolds number $\dot{E} a^{2} \varrho / \eta_{0}$ [16] (with $\varrho$ the density of the fluid) is sufficiently small (and provided the imposed rate of strain is not changing rapidly) the hydrodynamics of the particles and their ambient fluid is governed by the Stokes equation

$$
\eta_{0} \nabla^{2} \boldsymbol{u}=\nabla p
$$

Then the stresslet $\mathbf{S}_{i}$ is the sum of contributions due to tractions of Brownian motion, potential interaction and purely hydrodynamic interaction (superscripts $B, I$ and $H$ respectively) [14]

$$
\mathbf{S}=\mathbf{S}_{i}^{B}+\mathbf{S}_{i}^{I}+\mathbf{S}_{i}^{H} .
$$

The theory developed in this manner can be used to calculate the complex viscosity $\eta^{*}$ in a harmonically oscillating flow

$$
\eta^{*}=\eta^{\prime}(\omega)-i \eta^{\prime \prime}(\omega)
$$

where $\omega$ is the angular frequency.

The restriction to the two-body distribution function implies that only one relaxation process is involved. The pertinent relaxation time is the longest one since to deduce the radial distribution function one needs to integrate over all the positions of remaining $N-2$ particles in $V$. Apparently relaxations of a pair cannot occur until after the relaxation of the remaining $N-2$ particles in $V$. For one relaxation time $\lambda, \eta^{*}$ can be written as

$$
\eta^{*}=\eta^{\prime}(\infty)+\frac{\eta^{\prime}(0)-\eta^{\prime}(\infty)}{1+i \omega \lambda}
$$

In first order approximation [17] the $P_{2}$ is deformed from its equilibrium value

$$
P_{2}=n^{2} g\left[1-\frac{a^{2}}{2 D_{0}} \frac{\underline{\varrho} \cdot \dot{\mathbf{E}} \cdot \varrho}{\varrho^{2}} f \exp (i \omega t)\right] .
$$

Here $\varrho$ is the dimensionless radius vector $\boldsymbol{r} / a$ between two particles and $f$ is determined by the appropriate conservation equation for $P_{2}$ derived from eqs. (4) and (5). Analogously to eq. (10) $\mathrm{T}$ can be written as

$$
\mathbf{T}=\mathbf{T}^{H}+\mathbf{T}^{B}+\mathbf{T}^{I} .
$$

It turns out that for a dispersion with volume fraction $\phi[17]$

$$
\begin{aligned}
& \mathbf{T}^{H}=2 \eta_{0} \dot{\mathbf{E}}\left[1+\frac{5}{2} \phi+\frac{5}{2} \phi^{2}+\frac{15}{2} \phi^{2} \int_{2}^{\infty} J(\varrho) g \varrho^{2} d \varrho\right], \\
& \mathbf{T}^{I}=\frac{9}{20} \eta_{0} \phi^{2} \dot{\mathbf{E}} \int_{2}^{\infty} \varrho^{3}(1-A) \frac{d g}{d \varrho} f d \varrho, \\
& \mathbf{T}^{B}=\frac{9}{20} \eta_{0} \phi^{2} \dot{\mathbf{E}} \int_{2}^{\infty} \varrho^{2} W g f d \varrho .
\end{aligned}
$$

The functions $W(\varrho)$ and $A(\varrho)$ are determined by purely hydrodynamic interactions and $J(\varrho)$ represents particularly the near field hydrodynamic interactions. In a harmonically oscillating flow field $f$ is complex, so $f=f^{\prime}+i f^{\prime \prime}$. From eq. (4), applied for $P_{2}$, it follows that $f_{\omega \rightarrow 0}^{\prime \prime}=0, f_{\omega \rightarrow \infty}^{\prime}=0$ and

$$
f_{\omega \rightarrow \infty}^{\prime \prime \prime}=-\left[W-\varrho(1-A) \frac{d}{d \varrho}\left(\frac{V_{m f}}{k T}\right)\right] \frac{2 D_{0}}{a^{2} \omega}
$$

with the potential of mean force $V_{m f}$ given by

$$
V_{m f}=-k T \ln g(r) .
$$

Consequently

$$
\begin{aligned}
& \eta^{\prime}(0)=\frac{1}{6 \eta_{0}}\left(\dot{\mathbf{E}}^{-1}: \mathbf{T}\right) \\
& \eta^{\prime}(\infty)=\frac{1}{6 \eta_{0}}\left(\dot{\mathbf{E}}^{-1}: \mathbf{T}^{H}\right) \\
& G^{\prime}(\infty)=\omega \eta^{\prime \prime}(\infty) \\
& =\frac{9}{20} \eta_{0} \phi^{2} \frac{D_{0}}{a^{2}} \int_{2}^{\infty} \varrho^{2} g\left[W-\varrho(1-A) \frac{d}{d \varrho}\left(\frac{V_{m f}}{k T}\right)\right]^{2} \mathrm{~d} \varrho
\end{aligned}
$$


For $U=0$ the results are simple and given by Batchelor (and implicitly for $G^{\prime}(\infty)$ ) [14]

$$
\begin{aligned}
& \eta^{\prime}(0)(U=0)=\eta_{0}\left(1+\frac{5}{2} \phi+6.2 \phi^{2}\right), \\
& \eta^{\prime}(\infty)(U=0)=\eta_{0}\left(1+\frac{5}{2} \phi+5.2 \phi^{2}\right), \\
& G^{\prime}(\infty) \approx 3.8 \frac{D_{0}}{a^{2}} \eta_{0} \phi^{2} .
\end{aligned}
$$

Thus

$$
\lambda(U=0)=\frac{\eta^{\prime}(0)-\eta^{\prime}(\infty)}{G^{\prime}(\infty)} \approx 0.27 \frac{a^{2}}{D_{0}} .
$$

The potential $U$ of hard sphere repulsion makes the coefficients of $\phi^{2}$ dependent on $\phi[15]$. For $\phi=0.46$ (the volume fraction of the measured samples) we read from the table and figures in [15]

$$
\begin{aligned}
& \eta^{\prime}(0)=\eta_{0}\left(1+\frac{5}{2} \phi+18.4 \phi^{2}\right), \\
& \eta^{\prime}(\infty)=\eta_{0}\left(1+\frac{5}{2} \phi+7.4 \phi^{2}\right), \\
& G^{\prime}(\infty)=88 \frac{D_{0}}{a^{2}} \eta_{0} \phi^{2}, \\
& \lambda=0.125 \frac{a^{2}}{D_{0}} .
\end{aligned}
$$

Between $\phi=0.35$ and $\phi=0.60$ the coefficient of $a^{2} / D_{0}$ in $\lambda$ varies between 0.16 and 0.08 while $\eta^{\prime}(0)$ increases by an order of magnitude. This weak dependence of $\lambda$ on $\phi$ and $U$ reflects the physical significance of $\lambda$ in these theories (a linear phenomenon): $\lambda$ is determined by the diffusion time of a particle over a certain distance smaller than the particle radius which is hardly influenced by the potential interactions. By contrast the energy dissipated and stored during such a displacement is stronger dependent on interactions.

\section{Experimental}

\subsection{Samples}

The colloidal dispersions used were prepared according to the method described by van Helden [18]. The dispersions consist of amorphous silica particles which are made lyophilic by grafting octadecyl alcohol to the surfaces. The particles are dispersed in cyclohexane. By measuring the diffusion coefficient in a diluted sample one finds the fluid-dynamic or Stokes radius from $D_{0}=k T / 6 \pi \eta_{0} a$. The samples are labelled SJ 18 $\left[a_{\text {Stokes }}=(76 \pm 2) \mathrm{nm}\right]$ and SP23 $\left[a_{\text {Stokes }}=(28 \pm 2) \mathrm{nm}\right]$. Two samples were prepared, each having a volume fraction close to 0.46 .

\subsection{Rheological methods}

The dynamic viscosities were determined with four single torsion pendulums operating in the frequency range $80 \mathrm{~Hz}$ to $2500 \mathrm{~Hz}$ [19] and with a nickel tube torsion resonator in the frequency range $4 \mathrm{kHz}$ to $170 \mathrm{kHz}[20]$. The measuring principle is based on the determination of the resonance frequencies and bandwidths of the resonator immersed in air and subsequently in the liquid under investigation. The complex viscosity can be deduced from the differences in the frequencies and bandwidths as measured in the two media. Linearity of the dynamic viscosity was checked by measuring at different values of the maximum strain $\left(E_{\max }<0.01\right.$ and $\left.\dot{E}_{\max }<100 \mathrm{~s}^{-1}\right)$. The measurements were made at a temperature of 25.0 $\pm 0.1^{\circ} \mathrm{C}$.

\section{Results and discussion}

The linear viscoelastic behaviour of a system which is purely viscous for $\omega \rightarrow 0$ and viscoelastic for $\omega \rightarrow \infty$ [6] can be described by the complex viscosity

$$
\eta^{*}=\eta^{\prime}(\infty)+\sum_{i} \frac{\eta_{i}}{1+i \omega \lambda_{i}}
$$

where $\lambda_{i}$ is a relaxation time and $\eta_{i} / \lambda_{i}$ is a relaxation strength. If one assumes that all the relaxation times are proportional to $a^{2} / D_{0}(26,30)$ it becomes feasible to plot $\eta^{*}$ as a function of $a^{2} \omega / D_{0}$. In figure 1 the results are shown together with the theoretical predictions. The first conclusion which can be drawn is that more than one relaxation time is present (as expected); this is evident from the asymmetry of $\eta^{\prime \prime}$ as a function of $\omega$. Further it is observed that the measurements for $a=28 \mathrm{~nm}$ and $a=76 \mathrm{~nm}$ roughly coincide. This supports the deduction that the observed relaxations are proportional to $a^{3}$, which is typical for relaxation behaviour as treated in section 2. The discrepancies between the two sets of measurements (notably for $\eta^{\prime \prime}$ ) can be ascribed to uncertainties in $a( \pm 2 \mathrm{~nm})$ and $\phi$ $( \pm 0.02)$ and concerning the identity of the sterical interactions of the different sized particles.

The straightforward interpretation of the theory is that $D_{0}=k T / 6 \pi \eta_{0} a$, where $\eta_{0}$ is the viscosity of the continuous phase. It is, however, tempting to replace $\eta_{0}$ by another viscosity $\eta_{s}$ so that the relevant diffusion coefficient can be interpreted as the short time selfdiffusion coefficient [11]. Such a viscosity cannot be substituted unequivocally since the ambient of a sphere changes as a function of frequency. One would expect here values of $\eta_{s}$ between 7 and $13 \mathrm{mPa}$ (for 


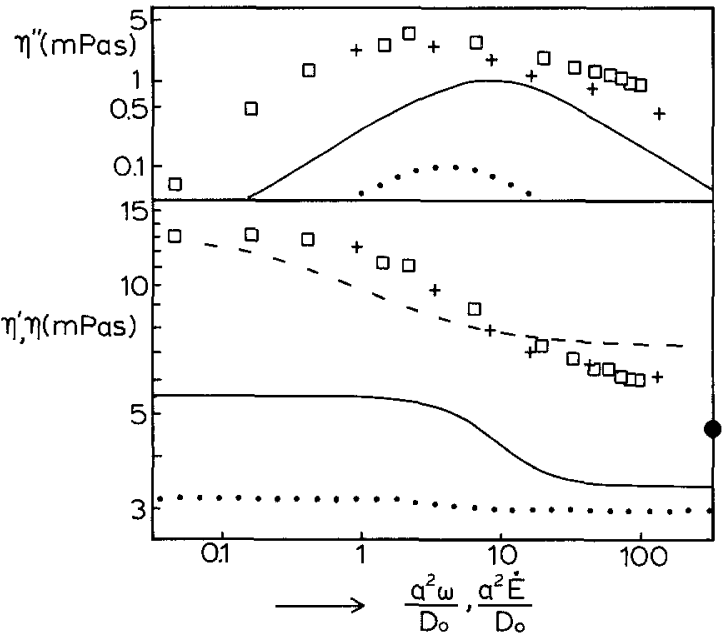

Fig. 1. Measured dynamic viscosities $\eta^{\prime}$ and $\eta^{\prime \prime}$ as a function of $a^{2} \omega / D_{0}$. Volume fraction of the two samples is $\phi \approx 0.46$. + - sample SJ 18 with mean particle radius $a=76 \mathrm{~nm}$, $\square-$ sample SP23 with mean particle radius $28 \mathrm{~nm}$. Theoretical predictions according to Batchelor $[14](\cdots \cdots)$ and Russel and Gast [15] (—). Beenakker's [21] calculations leads to - The steady shear viscosity $\eta$ as a function of $a^{2} \dot{E} / D_{0}$ as found by de Kruif [10] is indicated by (--- ). It should be noted that the theoretical predictions of [14] and [15] are not given explicitly but are calculated by us using eq. (26)

$\eta^{\prime}(\infty)$ and $\eta^{\prime}(0)$ respectively). The latter interpretation of $D_{0}$ is more consistent with the deduction in section 2 that the theory predicts the longest relaxation time. In addition it is more consistent with preliminary measurements which revealed that the relaxation times increase more strongly with $\phi$ than can be accounted for by the theoretical prediction for the longest relaxation time with $D_{0}=k T / 6 \pi \eta_{0} a$. The magnitudes of $\eta^{\prime}(0)-\eta^{\prime}(\infty)$ and $\eta_{\max }^{\prime \prime}$ predicted by Russel and Gast [15] are in agreement with our measurements, taking into account that the effect of only one relaxation time has been calculated. Those of Batchelor [14] are so small that one doubts whether even a large number of relaxation times of this type can explain the measurements.

Beenakker [21] combined the method of reflections (which accounts for the many-body-hydrodynamics) with available results for $g(r)$ to calculate the viscous contribution to the stress in the linear deformation region but in the high frequency (only hydrodynamics) limit. His calculation must thus be compared with $\eta^{\prime}(\infty)$. From figure 4 in [21] we read $\eta^{\prime}(\infty) / \eta_{0}=5.3$ and so $\eta^{\prime}(\infty) \cong 4.7 \mathrm{mPa}$ s. This value fits in with our result reasonably well, taking into account error ranges, see figure 1.

The question remains if any other effects can explain the observed phenomena. Given the sterical stabiliza- tion one might expect elasticity to arise from sterical interaction. It is hard to understand, however, how this interaction can give relaxation times proportional to $a^{3}$ whereas one would also expect linear effects for much smaller range of $E$ and $\dot{E}$ than the linear ranges covered in the present experiments (section 3 ).

In polymer rheology $\eta^{\prime}(\omega)$ is often found empirically to be close to the steady-state shear viscosity $\eta(\dot{E}=\omega)$. The equality

$$
\mid \eta^{*}(\omega)=\sqrt{\eta^{\prime 2}(\omega)+\eta^{\prime 2}(\omega)}=\eta(\dot{E}=\omega)
$$

corresponds to the Cox-Merz rule [22]. In figure 1 the non-linear behaviour of $\eta(\dot{E})$ [10] for $\phi=0.46$ is also plotted schematically. Obviously the $\left|\eta^{*}\right|(\omega)$ does not coincide with $\eta(\dot{E}=\omega)$. Conceptually in general there are also no fundamental reasons for such a coincidence since steady shear behaviour, unlike linear behaviour, can display a significant departure from equilibrium structures.

Pursuing the interpretation of the longest relaxation time $\lambda_{1}$, eq. (30), one can expect departure from linear behaviour if during the typical time $\dot{E}^{-1}$ of a flow a strain $E$ is produced so large that the relative displacement of the spheres is such that the equilibrium structure cannot be restored during that time. Consequently for $\dot{E}^{-1}>\lambda_{1}$ non-linear behaviour will occur. This prediction is also corroborated by the present measurements. In summary the preceding observations lead us to conclude that there is strong evidence that we have measured relaxation pertaining to thermodynamic forces.

\section{References}

1. Schoukens G, Mewis J (1978) J Rheol 22:381

2. Mitaku S, Oktsuki T, Okano K (1980) Jap J Appl Phys 19:439

3. Benzing DW, Russel WB (1981) J Colloid Interf Sci 83:163, 178

4. Buscall R, Goodwin JW, Hawkins MW, Ottewill RH (1982) J Chem Soc, Faraday Trans I 78:2873, 2889

5. Lindsay HM, Chaikin PM (1982) J Chem Phys $76: 3774$

6. Blom C, Mellema J, Lopulissa JS, Reuvers AJ (1984) Colloid \& Polym Sci 262:397

7. Hoffman RL (1972) Trans Soc Rheol 16:155

8. Hoffman RL (1982) Adv Colloid Interface Sci 17:161

9. Willey SJ, Macosko CW (1978) J Rheol 22:525

10. de Kruif CG, van lersel EMF, Vrij A, Russel WB (1985) J Chem Phys 83:4717

11. de Kruif CG, Jansen JW, Vrij A (1985) Intern Symp Phys Complex and Supermolecular Fluids: Colloids, Micelles and Microemulsions, Clinton, NJ, June 1985, to be published

12. Oosterbroek M, Mellema J, Lopulissa JS (1981) J Colloid Interface Sci 84:14, 27 
13. Blom C, Mellema J (1984) J Disp Sci Techn 5:193

14. Batchelor GK (1977) J Fluid Mech 83:97

15. Russel WB, Gast AP (1986) J Chem Phys $84: 815$

16. Batchelor GK (1967) An Introduction to fluid dynamics. p. 248, Cambridge University Press

17. Russel WB (1984) J Chem Soc, Faraday Trans II 80:31

18. van Helden AK, Jansen JW, Vrij A (1981) J Colloid Interface Sci 81:354

19. Blom C, Mellema J (1984) Rheol Acta 23:98

20. Oosterbroek M, Waterman HA, Wiseall SS, Altena EG, Mellema J, Kip GAM (1980) Rheol Acta 19:497

21. Beenakker CWJ (1984) Physica A 128:48

22. Bird RB, Armstrong RC, Hassager O (1977) Dynamics of polymer liquids, vol 1, p 194, John Wiley \& Sons, New York

(Received February 13, 1986)
Authors' adresses:

Dr. J. Mellema, C. Blom

Rheology Group, Department of Applied Physics

Twente University of Technology

Postbus 217

NL-7500 AE Enschede

Dr. C. G. de Kruif*), A. Vrij

Van't Hoff Laboratory for Physical and Colloid Chemistry University of Utrecht

Padualaan 8

NL-3584 CH Utrecht

*) To whom correspondence should be addressed. 